\title{
Concurrent fMRI demonstrates propagation of TMS effects across task-related networks
}

Lifu Deng ${ }^{1,8}$, Olga Lucia Gamboa ${ }^{1,2}$, Moritz Dannhauer $^{3}$, Anshu Jonnalagadda ${ }^{1}$, Rena Hamdan $^{3}$, Courtney Crowell ${ }^{1,2,4}$, Tory Worth ${ }^{1,8}$, Angel V. Peterchev ${ }^{2,3,5,6}$, Marc A. Sommer $^{1,3,7,8}$, Roberto Cabeza ${ }^{1,8}$, Lawrence G. Appelbaum ${ }^{9}$, Simon W. Davis ${ }^{1,4,8}$

${ }^{1}$ Department of Psychology \& Neuroscience, Duke University, Durham, NC 27708, United States of America.

${ }^{2}$ Department of Psychiatry and Behavioral Science, Duke University School of Medicine, Durham, NC 27708, United States of America.

${ }^{3}$ Department of Biomedical Engineering, Duke University, Durham, NC 27708, United States of America.

${ }^{4}$ Department of Neurology, Duke University School of Medicine, Durham, NC 27708, United States of America.

${ }^{5}$ Department of Electrical \& Computer Engineering, Duke University, NC 27708, United States of America.

${ }^{6}$ Department of Neurosurgery, Duke University School of Medicine, NC 27708, United States of America.

${ }^{7}$ Department of Neurobiology, Duke University School of Medicine, NC 27708, United States of America.

${ }^{8}$ Center for Cognitive Neuroscience, Duke University, NC 27708, United States of America.

${ }^{9}$ Department of Psychiatry, University of California, San Diego, CA 94143, United States of America

Correspondence should be addressed to Simon W. Davis (simon.davis@duke.edu)

\section{ACKNOWLEDGMENTS}

We thank Lamont Conyers and Jennifer Graves for extensive MRI support, and all subjects for their participation. This study was supported by the National Institute of Health, Brain Initiative 1RF1MH114253, R21-AG058161, and K01-AG053539.

\section{CONFLICT OF INTEREST}

A. V. Peterchev has received research funding, travel support, patent royalties, consulting fees, equipment loans, hardware donations, and/or patent application support related to magnetic stimulation from Rogue Research, Tal Medical/Neurex, Magstim, MagVenture, Neuronetics, BTL Industries, and Advise Connect Inspire. 


\section{ABSTRACT}

2 Transcranial magnetic stimulation (TMS) has become an important technique in both scientific and clinical practices, and yet our understanding of how the brain responds to TMS is still limited. Concurrent neuroimaging during TMS may bridge this gap, and emerging evidence suggests widespread that modulatory effects of TMS may be best captured through changes in functional connectivity between distributed networks, rather than local changes in cortical activity. However, the relationship between TMS stimulation parameters and evoked changes in functional connectivity is unknown. In this study, 24 healthy volunteers received concurrent TMS-fMRI while performing a dot-motion direction discrimination task. An MR-compatible coil was used to apply trains of three pulses at $10 \mathrm{~Hz}$ rTMS over the primary visual cortex (V1) at the onset of the dot stimuli with four levels of stimulation intensity $(20 \%, 40 \%, 80 \%$, and $120 \%$ of resting motor threshold, RMT). Behavioral results demonstrated impairment of motion discrimination at $80 \%$ RMT. FMRI results yielded three findings. First, functional connectivity between visual and non-visual areas increased as a function of rTMS intensity. Second, connectivity within the visual network was positively associated with motion accuracy, while the connectivity between visual and non-visual regions was negatively associated with motion accuracy. Lastly, we found that reductions in the similarity between functional and structural connectivity associated with increasing TMS intensity were constrained to the visual network.

19 These findings demonstrate spatially dependent nonlinear effects of TMS intensity on brain

20 functional connectivity that proceed beyond the site of stimulation and influence associated 21 behavior.

\section{INTRODUCTION}

23 Transcranial magnetic stimulation (TMS) has been a valuable tool in both cognitive and clinical

24 neuroscience because it allows for the examination of specific neurocognitive mechanisms and 25 the development of effective therapies based on these mechanisms. Ideally, the understanding 26 of mechanisms should precede clinical application, but even the simple dose-response 27 relationships of TMS are largely unknown outside of the motor cortex. Multiple lines of evidence 28 in animal models and human studies suggest that TMS induces complex changes in activity 29 across broad networks of cortical regions, demonstrating clear evidence of polysynaptic effects 30 (Wang and Voss, 2015; Cocchi et al., 2016; Alekseichuk et al., 2019; Beynel et al., 2020b). In 
31 line with these empirical findings, a recent systematic review of TMS on resting state networks (Beynel et al., 2020a) found that while network-level effects were common in the literature, these effects did not follow the typical frequency-dependent heuristic that helps to predict the local effect of TMS modulation (Luber and Deng, 2016). As such, it can be inferred that networklevel modulatory effects depend on not only the parameters of stimulation but also the functional interactions between the stimulated and connected regions.

TMS during concurrent neural recordings has become an exciting technological development that may expand the repertoire of addressable questions by leveraging the rich spatial and temporal information afforded by different imaging methods. Previous studies utilizing concurrent TMS and fMRI are often focused on resting-state brain activity without the presence of cognitive tasks. In such studies, stimulation of primary sensory or motor cortices frequently is reported to exert feed-forward influences on functionally relevant regions, such as the frontal eye fields (Cocchi et al., 2016; Castrillon et al., 2020). Similarly, concurrent TMS during electroencephalographic (EEG) recordings has shown that single-pulse TMS to the dorsal attention network results in widespread activation of that same network (Ozdemir et al., 2020). There is also evidence that these intra-network effects are facilitated by structural connectivity (Momi et al., 2021a), suggesting that stimulation propagates between brain regions that are structurally connected to the stimulation site.

A lingering question is the relevance between these TMS-driven dynamics and cognition, as the effects of TMS has been shown to depend on the cognitive state (Silvanto et al., 2018). For example, when rTMS is applied to motion selective cortex (MT+) during a motion discrimination task, performance is hindered when attention is focused on motion but enhanced when attention is focused on other visual attributes (Brascamp et al., 2010; Zinchenko et al., 2021). Relatedly, connectivity can depend on brain state during motion perception, based on evidence that frontal and occipital connectivity is only present when participants are aware of apparent motion (Sanders et al., 2014). In addition, the timing of stimulation relative to the onset of visual motion strongly determines the influence on performance and EEG responses (Gamboa Arana et al., 2020), implying the different cognitive components affected. Because attention-driven activity during motion-perception tasks is influenced by gating between primary visual cortex and MT+ (Stephan et al., 2008), a network approach may better explain the effects of TMS than focusing on a single region.

Building on past studies on concurrent TMS-neuroimaging, we sought characterize the

63 effects of TMS on network-level functional connectivity (FC) due to different intensities during 64 visual motion perception. Graph theoretical analyses were used to depict the organization of 
65 functional sub-systems and how TMS influenced communication between these systems. Three

66 specific goals were pursued. First, this study investigated whether TMS affected global or local

67 FC, and whether the effects were constrained to the network related to the task. Secondly, this

68 study examined the effects of $\mathrm{FC}$ on motion discrimination task performance to better 69 understand how changes in FC mediate behavior. Lastly, structural connectivity (SC) was used

70 to explore how TMS alters the functional network relative to the underlying architecture. This 71 analysis combines structural, functional, and behavioral information to further the understanding 72 of the impacts of TMS on global brain network and behavior.

\section{METHODS}

\section{$75 \quad 2.1$ Subjects}

A total of 27 healthy volunteers (16 females, mean age $=23.7$ years, $S D=2.78$ ) participated in this study. At the beginning of the first visit, all participants were informed about the experimental procedures and were screened for contraindications to MRI and TMS. Subsequently, written informed consent was obtained from each participant. All participants were right-handed according to the Edinburgh Handedness Inventory (Oldfield 1971), had normal or corrected-to-normal vision, and did not have a history of neurological or psychiatric diseases. Participants received $\$ 20$ per hour for taking part in this study. Procedures in this study conformed to the Declaration of Helsinki and were approved by the Institutional Review Board of Duke University. Data from three participants were excluded from the study as they declared falling asleep during the MRI session. As such, the final dataset analyzed for this study

\section{$87 \quad 2.2$ Experimental design}

Participants in this study took part in two experimental sessions. During the first session, participants were provided with screening questionnaires and were administered drug and pregnancy tests to check for eligibility. Resting motor threshold (RMT) was then acquired and

91 subjects were briefly familiarized with the motion discrimination task by performing 1-2 minutes

92 of practice trials. During the second session, participants first practiced the motion 
discrimination task in a staircase fashion inside a mock MRI scanner that mimics the environment of real MRI scanner (projection system, response box, recorded scanner sound, etc.). Before starting the fMRI-TMS data collection, participants were positioned in the bore of the MRI scanner and performed one additional staircase of the motion discrimination task to determine the threshold coherence level used in the subsequent fMRI-TMS task, as described in Section 2.3.1. After fMRI-TMS data acquisition, additional structural and diffusion weighted images (DWI) were acquired.

100
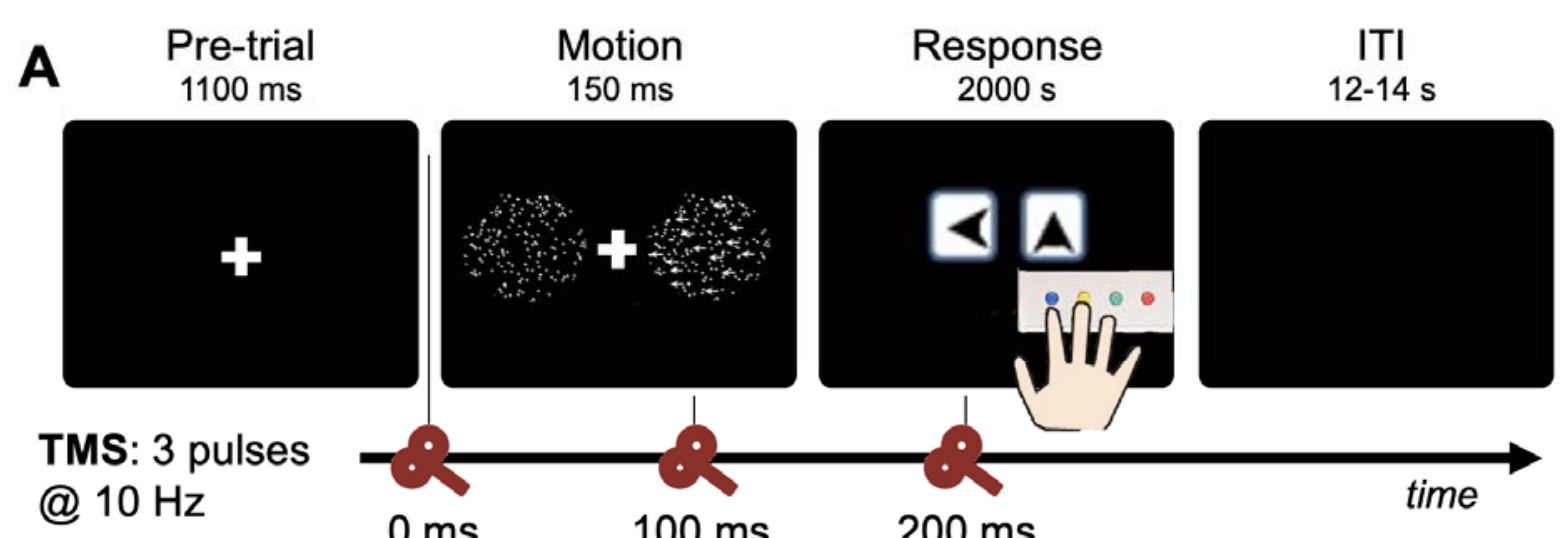

TMS: 3 pulses

@ $10 \mathrm{~Hz}$

$0 \mathrm{~ms}$

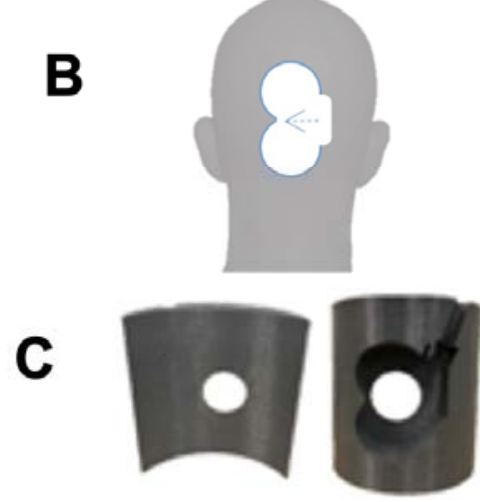

$100 \mathrm{~ms}$
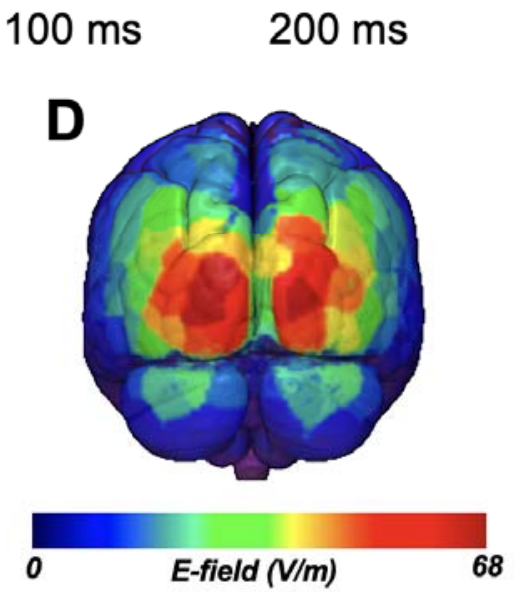
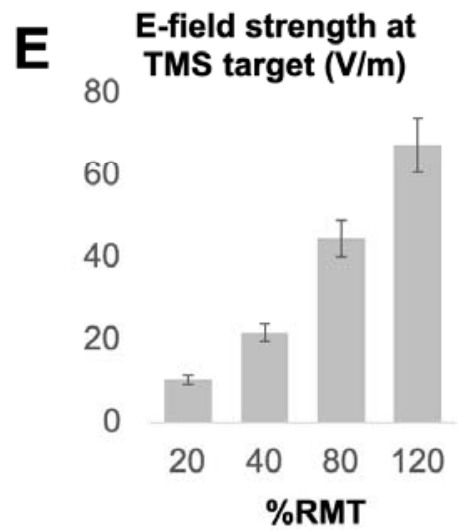

Figure 1. Experimental design. (A) Illustration showing still frames of the visual stimulus along with three TMS pulses (orange markers) presented at $10 \mathrm{~Hz}$ at the start of stimulus presentation. (B) Coil orientation and stimulation site over Oz as defined in the 10-20 EEG System. (C) 3D-printed headrest used in the MRI scanner to position the TMS coil over the stimulation site on Oz. Four different intensities were used: Oz stimulation at 20,40, 80 and 120\% RMT as the main tests and vertex stimulation at 120\% RMT as control. (D) Simulation of the TMS electric field (E-field) strength induced within each cortical node, averaged over all participants. (E) Simulated E-field strength (mean \pm standard deviation) at the target cortical node for each Intensity level across participants. 


\subsection{Procedures}

\section{2.3.1 Motion perception task}

112 Stimuli were generated using MATLAB (Mathworks, Natick, MA, USA) and the Psychtoolbox 113 extension (http://www.psychtoolbox.org). Each trial started with a white fixation-cross shown in 114 the middle of the screen on a black background (see Fig. 1A). After $530 \mathrm{~ms}$, white dots (5 pixels 115 each, dot density: $1.41 \mathrm{dots} / \mathrm{deg}^{2}$, dot- speed: $7.4 \%$ s) were presented for $150 \mathrm{~ms}$, moving at a 116 pre-determined coherence level (see next paragraph) either upwards or to the left within a 117 circular window on the upper right quadrant of the screen. The center of the dot field was 118 located at $8^{\circ}$ from the fixation cross and extended $12^{\circ}$ in diameter. Stimuli were projected onto a 119 mirror placed above the scanner head coil using a rear projection system. The projector refresh 120 rate was $60 \mathrm{~Hz}$ and viewing distance between the mirror and the projector screen was $70 \mathrm{~cm}$. 121 When needed, vision was corrected using MRI-compatible lenses matching a participant's 122 prescription. Responses were recorded via a fiber-optic response box (Resonance Technology, 123 Inc). Participants had up to $2 \mathrm{~s}$ to indicate if dot motion was to the left using the right index finger 124 or upwards using the right middle finger. After each response, a cross colored either green 125 (correct response) or red (incorrect response) was shown during $1 \mathrm{~s}$ to provide feedback. 126 Participants were instructed to perform the task as accurately and as quickly as possible, with 127 an emphasis on accuracy.

128 Before starting the TMS-fMRI data collection during the second session, subjects performed 129 a final practice version of the motion perception task within the bore of the magnet comprising 130120 trials in which dot coherence varied from $0 \%$ coherence to $100 \%$ coherence according to a 131 staircase procedure. Data from this practice session was assessed online to ascertain an 132 individualized coherence value to use in the main task. As in our previous work with this 133 paradigm (Gamboa Arana et al., 2020; Gamboa et al., 2020), the calculation was done by 134 means of a generalized linear model (MATLAB's glmfit function with 'binomial' option) that fit a 135 sigmoid function to the signed (+1/-1 due to dot motion direction) trial coherences and individual 136 correct/incorrect responses to find a particular coherence where $70 \%$ of the training trials were 137 correct.

\section{2.3.2 Concurrent TMS-MRI}

139 After the practice task in the mock MRI scanner, subjects were transferred to the MRI scanner 140 room. Participants wore a tightly fitting swimsuit cap where the stimulation points were marked. 141 The main stimulation target was centered over Oz as defined in the 10-20 EEG system, which is 
142 located $10 \%$ of the distance between the inion and nasion (approximately $3 \mathrm{~cm}$ above the inion 143 in most adults, see Fig. 1B) (Ishikawa et al., 2011). The coil was placed inside a custom 144 headrest that was 3D printed from semi-flexible material (nGen_FLEX) to be comfortable for the 145 subject and maintain the TMS coil position on the head during pulses (Fig. 1C). The headrest 146 was specially designed for each stimulation target so that the center of the coil was tangential to 147 the stimulation point and the induced electric field was perpendicular to the midline, while the 148 cable-side of the coil was on the right hemisphere. Positioning was adjusted both before the 149 scan using markers on the fitted swim cap and was verified after the experiment with the use of 150 four MR-sensitive fiducial markers placed over the borders of the major and minor semi-axes of 151 the coil. Cushions were used to reduce head motion inside the scanner and participants were 152 informed about the importance of minimizing head motion during task performance. Participants wore earplugs to attenuate scanner noise and the TMS clicking sound.

A burst of three biphasic TMS pulses at $10 \mathrm{~Hz}$ delivered at motion onset was applied over the stimulation site using a MagPro R30 magnetic stimulator connected to an MRI-compatible TMS figure-of-eight coil (MRi-B91 Air-cooled, MagVenture, Farum, Denmark). Stimulation over the visual area was delivered at $20 \%, 40 \%, 80 \%$ and $120 \%$ of the participant's RMT, presented in pseudo-randomized order in each of the runs. The task was presented in the form of a slow event-related design, such that the duration of each trial was $15 \mathrm{~s}$. The TMS device, the presentation computer, and the MR acquisition were synchronized via an Arduino board (Fig. 2A), such that the TMS pulses were delivered reliably in between slice acquisitions. Furthermore, timing of the MR acquisition rate (30 slices every 2 seconds, or $15 \mathrm{~Hz}$ ) and TMS frequency $(10 \mathrm{~Hz})$ allowed us to place the TMS pulses consistently inferior (slice 4/30, roughly at the level of the lower cerebellum) and superior (slice 28/30) locations, ensuring that artifacts associated with the TMS pulse were spaced at least 4-6 slices away from the principle regions of interest (i.e., visual cortex, Fig. 2B); Arduino code used to time pulses can be found online in our public repository (https://github.com/ElectricDinoLab). The task was divided into five runs, with each run containing 32 trials (8 trials for each intensity), giving a total 40 trials per Intensity over the course of an fMRI session. As each trial comprised three $10 \mathrm{~Hz}$ pulses, a total of 120 pulses were administered at each intensity. 


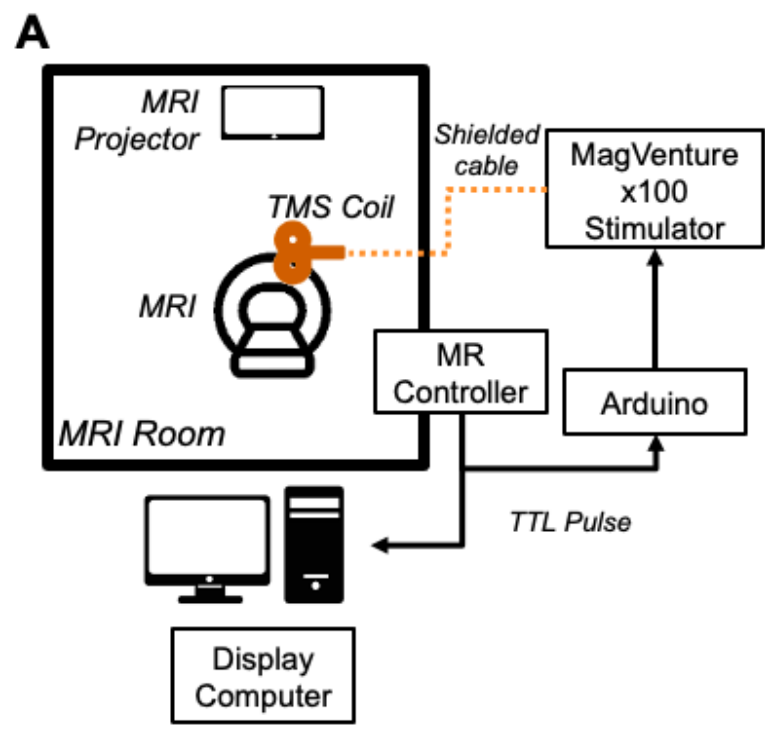

B

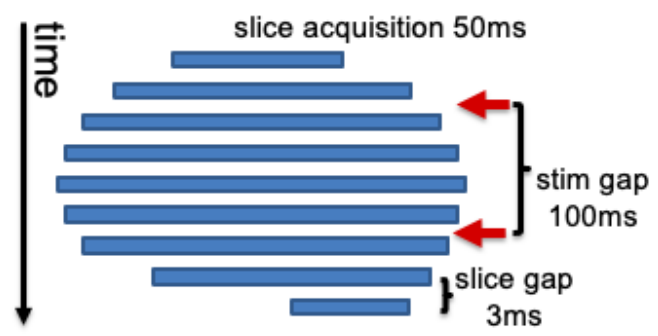

Figure 2. Concurrent TMS-fMRI. (A) A generalized wiring diagram. (B) Schematic of the timing of TMS pulses to avoid concurrent acquisition of data and TMS pulses.

\section{2.3.3 Resting motor threshold (RMT) measurement}

175 To determine the TMS intensities to use during the experiment, the resting motor threshold 176 (RMT) of the right first dorsal interosseous muscle (FDI) representation in the left primary motor 177 cortex was obtained in the first visit. During this procedure, biphasic single pulses were sent 178 with a MagPro R30 stimulator connected to a figure-of-eight coil (MCF-B65 cool coil, 179 MagVenture, Farum, Denmark) while being guided by a stereotaxic neuronavigation system 180 (Brainsight, Rogue Research, Canada). Surface electromyogram (EMG) was recorded from the 181 right FDI muscle by using disposable electrodes (Covidien / Kendall, 133 Foam ECG 182 Electrodes, Mansfield, USA) in a belly tendon montage. RMT was defined as the minimum 183 intensity at which a motor evoked potential (MEP) of $50 \mu \mathrm{V}$ average peak-to-peak amplitude 184 was elicited when the muscle was at rest (Conforto et al., 2004). The Motor Threshold 185 Assessment Tool software (MTAT 2.0) (http://www.clinicalresearcher.org) was used to 186 determine the RMT once a reliable stimulation site for the FDI motor 'hot spot' was found. The 
187 percentage of the maximum stimulator output and the realized peak coil current rate of change

$188(\mathrm{dl} / \mathrm{dt})$ values were registered to be used in the following session.

189 2.3.4 fMRI data acquisition \& analysis

190 Functional MR images were obtained with a 3T GE MRI system scanner with a 4-channel 191 transmit/receive head coil at the Brain Imaging and Analysis Center (BIAC) at Duke University 192 Medical Center. During the visual task an echoplanar imaging (EPI) sequence was used (320 193 volumes, 30 axial slices, repetition time/echo time $=1500 / 27 \mathrm{~ms}$; flip angle $=70$; FOV $=256$ $194 \mathrm{~mm}^{2}$; matrix size $=64 \times 64$; voxel size $=4 \times 4 \times 4 \mathrm{~mm}^{3}$; slice thickness $\left.=4 \mathrm{~mm}\right)$. The first $9 \mathrm{~s}(6$ volumes) of the fMRI record were discarded to allow for T1 equilibrium magnetization. Anatomical images for co-registration were collected using a T1-weighted MP-RAGE sequence (repetition time/echo time/inversion time $=6.972 / 3.01 / 400 \mathrm{~ms}$, flip angle $=11^{\circ}$ ).

Functional image processing proceeded first by removing slices affected by TMS pulses. This was necessary because, even though TMS pulses were timed to be delivered in between MR slice acquisitions, small errors in timing may have resulted in overlap between the TMS pulse and MR acquisition. We identified slices with a signal magnitude of $>2$ SD from the run mean and visually inspected them for the presence of the TMS artifact. These slices were replaced by temporal interpolation of the signal values of the same slice from the preceding and succeeding volumes. Functional images were then preprocessed using image processing tools, including 205 FLIRT (FMRIB's. Linear Image Registration Tool) and FEAT (FMRIB Expert Analysis Tool) from 206 FMRIB's Software Library (FSL, https://fsl.fmrib.ox.ac.uk/fsl/fslwiki/). Images were corrected for 207 slice acquisition timing, motion, and linear trend; motion correction was performed using FSL's 208 MCFLIRT, and six motion parameters estimated from the step were then regressed out of each 209 functional voxel using standard linear regression. Images were then temporally smoothed with a 210 high-pass filter using a $190 \mathrm{~s}$ cutoff and normalized to the MNI stereotaxic space. To remove 211 any additional artifacts associated with TMS pulses, spatiotemporal independent components 212 analysis (ICA, FSL's MELODIC) was used to identify and remove noise components occurring 213 in slices collected concurrent with the TMS pulse. White matter and CSF signals were also 214 removed from the data and regressed from the functional data using the same method as the 215 motion parameters. BOLD activations during the initial dot motion presentation were entered in 216 a standard GLM, using HRF-convolved trial regressors and their temporal derivatives. 


\subsubsection{Electric field modeling}

218 The direct effects of TMS are driven by the strength and spatial distribution of the electric field 219 (E-field) induced in the brain. Thus, simulation of the E-field induced by TMS in individual participants is increasingly recognized as an important step in spatial targeting of specific brain regions and forms a critical link between the externally applied TMS parameters and the neurophysiological response supporting cognitive operations (Peterchev et al., 2012; Gomez et al., 2020). To determine the E-field induced by TMS in the brain of each participant, simulations using T1 images and the finite element method in the SimNIBS software package (version 2.0.1, Thielscher et al., 2015) were conducted (Beynel et al., 2019; Beynel et al., 2020c; Beynel et al., 2021). Twenty-three subjects were included in the E-field analysis and head models were created using the SimNIBS mri2mesh pipeline, featuring five distinct tissue types: skin, skull, cerebrospinal fluid, gray matter, and white matter. The scalp, skull, and cerebrospinal fluid tissue compartments were modeled with isotropic conductivity values of $0.465,0.01$, and 1.654 $\mathrm{S} / \mathrm{m}$, respectively. For seventeen subjects, available DWI information was used to estimate anisotropic conductivities for the brain matter using the volume-normalized approach (Gullmar et al., 2010) which kept the geometric mean of the conductivity tensor eigenvalues for the gray and white matter equal to literature-based isotropic values of 0.275 and $0.126 \mathrm{~S} / \mathrm{m}$, respectively. DWI data could not be processed for six subjects, for which the latter isotropic conductivity values for gray and white matter were assigned to their corresponding computational tetrahedral elements. For nine subjects, 4 fiducial markers implemented with gel-filled stickers were attached on the active side of the TMS coil to image the location of the coil relative to the head using additional T1 scans. Based on the location of the fiducial markers, the mean (+/- standard deviation) shortest distance between the TMS coil center and the scalp was determined to be $3.8 \mathrm{~mm}(+/-3.4 \mathrm{~mm})$ across the nine subjects, and the coil plane deviated from the local scalp tangential plane by only $7.2^{\circ}\left(+/-3.2^{\circ}\right)$, confirming good coil placement. For fifteen subjects it was not possible to consistently image the fiducial markers. Instead, the target $\mathrm{Oz}$ scalp site was identified from the MRI data and head model's scalp surface, the coil center was extruded by $4 \mathrm{~mm}$ to account for the scalp-coil spacing, and the coil plane was oriented tangentially to the closest scalp surface location. The individual E-field was simulated for TMS coil current rate of change $(\mathrm{dl} / \mathrm{dt})$ of $1 \mathrm{~A} / \mu \mathrm{s}$. Leveraging the direct proportionality between the coil current and the

247 E-field strength, the latter was obtained by scaling the E-field solution for the various TMS 248 intensities based on the corresponding $\mathrm{dl} / \mathrm{dt}$ value computed from the Intensity setting according 249 to the formula $\mathrm{dl} / \mathrm{dt}=1.9308 \times \% \mathrm{MSO}-2.4018$. The formula was derived by linear regression 
of the $\mathrm{dl} / \mathrm{dt}$ values reported by the TMS device for Intensity settings as percentage of maximum stimulator output (\%MSO) for 5\% MSO steps averaged over three TMS pulses. Figures 1D and 1E present, respectively, a map of the cortical-node E-field strength at $120 \%$ RMT averaged across the subjects, and the E-field strength at the target cortical nodes across the four TMS

254 intensity levels.

255

256

257

258

259

260

261

262

263

264

265

266

267

268

269

270

271

272

273

274

275

276

277

278

279

280

281

282

\subsubsection{Network Analysis}

Functional connectivity (FC) network construction. A consistent parcellation scheme across all subjects and all modalities (DWI, fMRI) was used. Subjects' T1-weighted images were segmented using SPM12 (fil.ion.ucl.ac.uk/spm/software/spm12/), yielding a GM and white matter mask in the T1 native space for each subject. The entire GM was then parcellated into 397 ROls, each representing a network node by using a sub-parcellated version of the HarvardOxford Atlas (Fornito et al., 2010). Functional connection matrices representing task-related connection strengths were estimated using a correlational psychophysical interaction (cPPI) analysis. Briefly, the model relies on the calculation of a PPI regressor for each region, based on the product of that region's physiological time series and a task regressor convolved with the HDR, to generate a term reflecting the psychophysical interaction between the seed region's activity and the specified experimental manipulation. The psychological regressor was multiplied with two physiological time courses for regions $\mathrm{i}$ and $\mathrm{j}$, producing two psychophysiological interaction regressors, denoted as $\mathrm{PPI}_{\mathrm{i}}$ and $\mathrm{PPI}_{\mathrm{j}}$. The partial correlation $\left(\rho_{\mathrm{XY} \cdot \mathrm{z}}\right.$, where $\mathrm{X}=\mathrm{PPI}_{\mathrm{i}}$, $\mathrm{Y}=\mathrm{PPI} \mathrm{j}_{\mathrm{j}}$ ), was then computed as the partial correlation between two psychophysiological interaction regressors for a given pair of regions $i$ and $j$, controlling for the covariates (denoted as $z$, which includes the HDR-convolved task regressor and the BOLD time courses for regions $i$ and $j$ ); the six motion parameters, white matter signal, and CSF signal were also included as confounds.

Modularity. Functional sub-network community assignment was identified using modularity analysis (Reichardt and Bornholdt, 2006). A common whole-brain network was obtained by averaging all functional networks across participants and intensity levels. This common network was then thresholded to preserve $15 \%$ strongest connections and submitted to the modularity algorithm (gamma = 1). Based on module assignments, functional connections were classified into between-module connections (connecting regions in different modules) and within-module connections (connecting regions in the same module).

Structural connectivity (SC) network construction. Information on the structural connections based on diffusion tractography between each pair of regions in the data was assessed with a 
standard DWI processing pipeline used previously in our group (Davis et al., 2019). DWI data were analyzed using FSL (fsl.fmrib.ox.ac.uk) and MRtrix (mrtrix.org) software packages. Data were denoised, corrected with eddy current correction, and bias-field corrected. Constrained spherical deconvolution was used in calculating the fiber orientation distribution; after tracts were generated, they were filtered using spherical-deconvolution informed filtering of tractograms (SIFT; Smith et al., 2013). To generate the SC network, the value of SC was

289 defined as the number of streamlines connecting each pair of ROls.

\section{0}

291

292

293

294

295

296

297

298

299

300

301

302

303

304

305

\subsubsection{Statistical Analysis}

Statistical analyses were performed using MATLAB and RStudio. The effect of TMS intensity on behavior was assessed using repeated-measures ANOVA. The FC network of each participant under each rTMS intensity was first normalized into z-scores to further control for motion-induced confound. The between-and within-module FCs were then derived from the $z-$ scored network. The effect of TMS on FC within and between network was assessed using twoway repeated-measures ANOVA, in which the Type of FC (between/within) and the Intensity of TMS (20/40/80/120\% RMT) were defined as the two main effects. The relationship between connectivity and task performance was examined in mix effect regression model using the Ime4 package in $\mathrm{R}$, in which the dependent variable was the correct response rate. The random effect was specified as participant-specific intercept. Variables of interests, estimated as fixed effects, were TMS intensity, between-module and within-module FC of the visual module, as well as participant-level covariates such as sex, coherence of the visual stimuli, and resting motor threshold. The neural effects on performance were assessed using F-contrasts and Tcontrasts. Details about the design matrix and the contrasts can be found in Figure S1a and Figure S1b, respectively, in Supplementary Materials. 


\section{3. RESULTS}

\section{$309 \quad 3.1$ Behavioral Results}

310 The accuracy of motion discrimination task (leftwards vs. upwards motion) during concurrent 311 TMS-fMRI was analyzed to assess the behavioral effect of TMS targeting visual cortex. A one312 way repeated-measures ANOVA revealed significant differences in performance across TMS 313 intensity levels $\left(\mathrm{SS}_{\text {subjects }}=0.69, \mathrm{SS}_{\text {condition }}=0.04, \mathrm{~F}_{3,23}=2.93, p=0.039\right)$. Post hoc, uncorrected 314 comparisons between TMS Intensity conditions revealed that motion accuracy in the $80 \%$ RMT 315 condition (mean: 58\% $\pm 9 \%$ ) was significantly lower than $20 \%$ RMT (mean: $63 \% \pm 13 \%, p=$ 316 0.011), 40\% RMT (mean: 62\% $\pm 10 \%, p=0.024$ ), and 120\% RMT (mean: 61\% $\pm 9 \%, p=$ 317 0.047) conditions (Fig. 3A). No differences in discrimination accuracy were seen between the 318 highest (120\% RMT) and lowest (20\% RMT) stimulation intensities ( $p>0.05)$, suggesting that 319 the disruptive effects were attributable to a neural response in visual processing regions rather 320 than distraction-related confounds (sound, somatic sensations) associated with increasing TMS 321 intensity. Reaction times (Fig. 3B) were not significantly affected by the different TMS 322 intensities, though correct trials were significantly faster than incorrect trials (paired $t_{23}=5.33, p$ $323=0.0002)$.

324

325
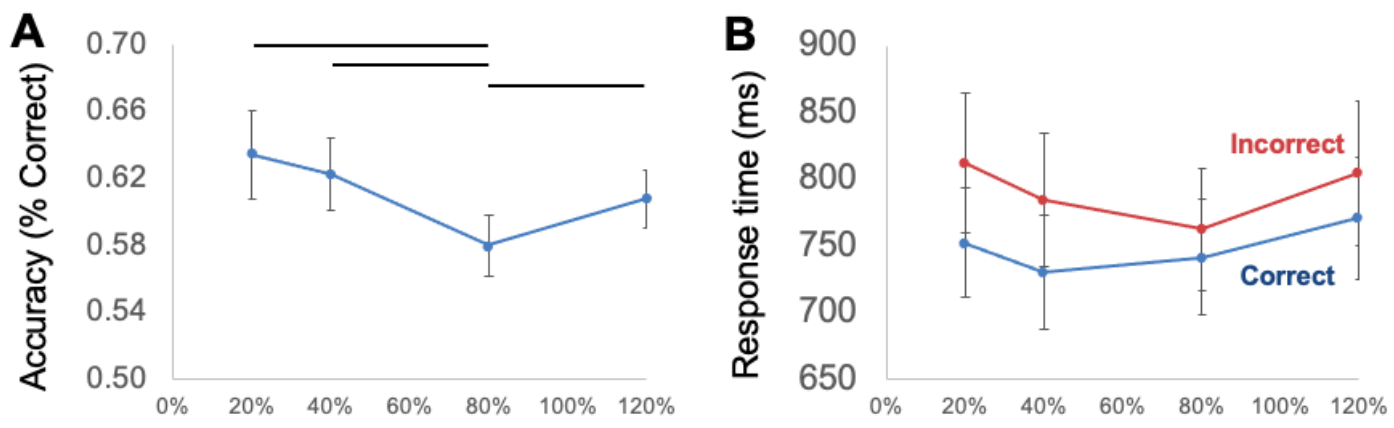
Figure 3. Behavioral results. Accuracy (A) and reaction times $(\boldsymbol{B})$ for all participants in the dot-motion perception task during TMS-fMRI. TMS selectively disrupted motion performance at $80 \%$ RMT, relative to all other stimulation intensities. In contrast, no effects of Intensity were seen on reaction time.

\subsection{Delineating task network nodes affected by TMS}

The present analysis focused on evaluating network-level, fMRI-based functional connectivity to characterize the effects of stimulation intensity. The network-level effects of TMS were examined on two scales: brain modules, and directly stimulated nodes in visual cortex. Stimulated nodes were defined using E-fields estimated within each individual, localized using fiducial markers placed on the TMS coil; E-fields were then scaled by individual RMT, and then voxelwise E-field values were averaged within a parcellated region of interest to identify the meaningful networks to establish reliable boundaries between local and global communities of regions, termed "modules" (also known as "subnetworks"), by using modularity analysis on the average FC matrix (averaging across subject, TMS intensity, and task success conditions). This data-driven modularity analysis parsed 397 ROls into four highly recognizable modules (Fig. S2), including (i) default mode network (DMN), (ii) somatosensory-motor network (SM), (iii) a visual-temporal network complex, and (iv) frontal-parietal control network (FPCN). To obtain a more accurate delineation of the visual system in the Visual-Temporal complex, an additional modularity analysis was performed on it, which in turn produced four subdivisions (Fig. S3); among these subdivisions, the two posterior subdivisions were combined into the Visual network (Vis.), and the two anterior, the Temporal network (Tmp.). The final 5-module assignment is shown in Figure 4A.

\subsection{TMS effects on functional connectivity}

\subsubsection{TMS effects on visual system functional connectivity}

Network-level TMS effects were first evaluated on the task-relevant regions. The 5-module community assignment (Fig. 4A) was used to define within-module FC (the average FC within

353 the same module) and between-module FC (the average connectivity between the given 354 module and other modules). For each of the five modules, an Intensity (\% RMT) by Type 355 (within/between module connectivity) repeated-measures ANOVA was performed. For all five 356 modules, the within-module FC was significantly stronger than the between-module FC (DMN: $357 \quad F_{1,21}=11.8, p=0.0023 ; S M: F_{1,21}=40.52, p=1.7 e-6$; Visual: $F_{1,21}=33.04, p=7.4 e-6$; Temporal: $\left.358 \mathrm{~F}_{1,21}=17.45, \mathrm{p}=3.6 \mathrm{e}-4 ; \mathrm{FPCN}: \mathrm{F}_{1,21}=17.23, \mathrm{p}=3.9 \mathrm{e}-4\right)$, suggesting that the data-driven 
modularity analysis successfully captured meaningful modules of the whole-brain functional network. For completeness, results for the larger Visual-Temporal complex are shown in Fig. S2 in the Supplementary Materials, which were similar to the findings for the Visual network.
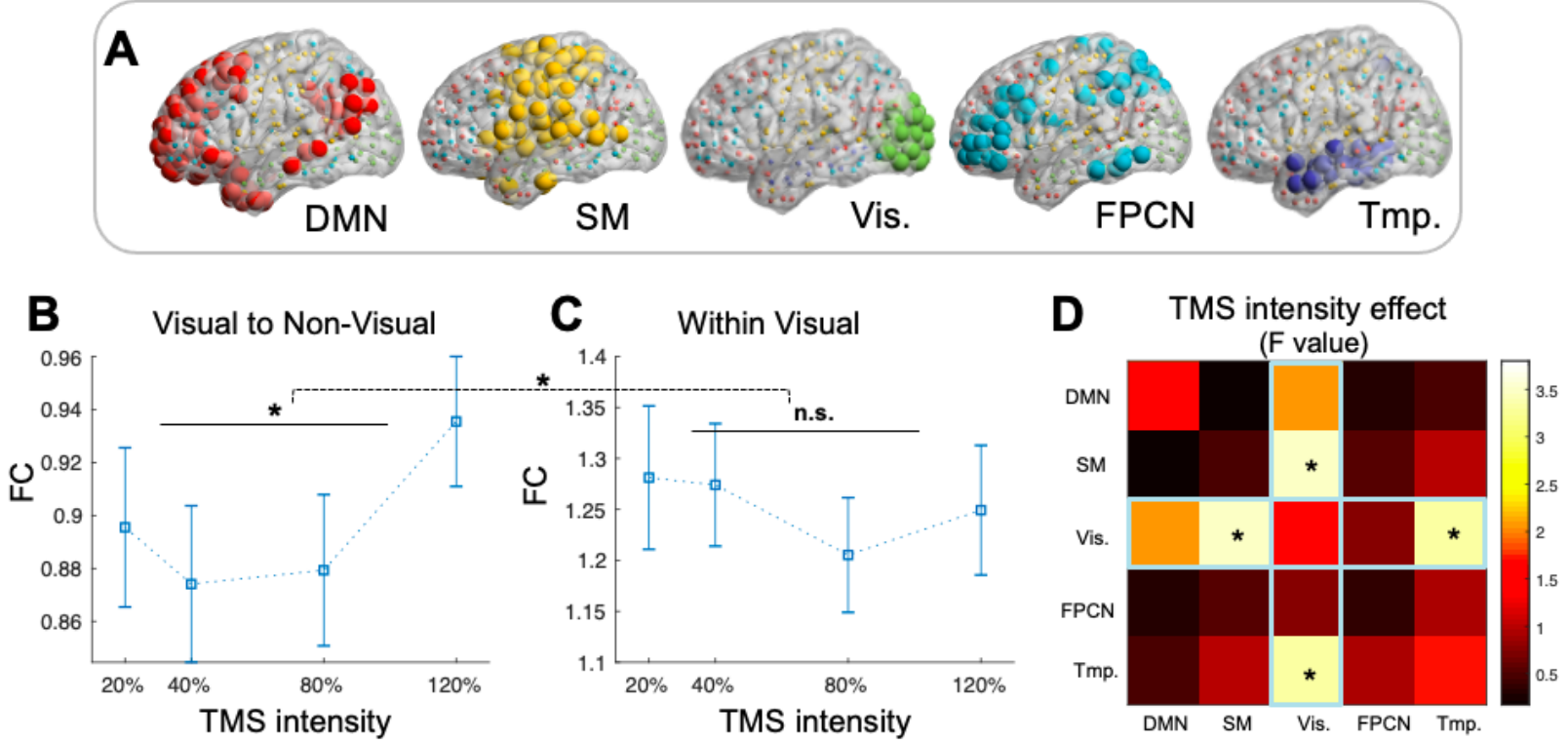

Figure 4. Within and between-module connectivity in response to TMS. (A) The 5 modules obtained from modularity analysis $(D M N=$ default mode network, $S M=$ somatosensory-motor network, Vis. = visual network, $F P C N=$ frontal-parietal control network, Tmp. = temporal network). (B) and (C) The effects of TMS intensity on visual network FC (z-scored); dashed horizontal brace indicates the Intensity $x$ Type interaction; horizontal solid lines indicate the main effects of Intensity. (D) The effects of TMS intensity (expressed as F-values) on the FC between different modules. Error bars indicate standard error of the mean (SEM). Asterisks (*) indicate $p<0.05$.

Crucially, a significant Intensity $x$ Type interaction was found uniquely in the Visual network $\left(F_{3,69}=3.99, p=0.011\right.$, Fig. 4B, 4C) but not in the other modules (all $p>.05$ ), suggesting that the TMS may have a type-specific effect on the functional communication of the targeted visual system. The effects of TMS intensity on these two types of FC were further examined in two separate one-way repeated measure ANOVAs, where the intensity effect was significant for between-module connectivity (i.e., 'Visual to Non-Visual', $F_{3,69}=2.88, p=0.039$, Fig. 4B) but insignificant for within-module connectivity (i.e., 'Visual to Visual', $F_{3,69}=1.69, p=0.178$, Fig. 4C). An exploratory analysis suggested that such significant modulation of between-module FC seemed to be driven by the connections with SM and Temporal modules; both findings are consistent with the idea that the effects of increasing TMS elicited greater network activity due to increasing motor (SM) or auditory (Temporal) sensation. No significant effect of TMS intensity 
existed between other modules (Fig. 4D). In sum, these results showed that TMS modulated FC of the targeted functional system.

The above results support the idea that intensity effects can be seen at the level of the stimulated network, but a more rudimentary concern might be that these same effects can also be seen at the level of the directly stimulated region. To examine this possibility, two nonoverlapping groups of visual ROls were further investigated: 1) three ROls in the left occipital pole that were closest to the TMS coil (Group 1, "directly-stimulated visual cortex"), which also received visual inputs from the right visual field and, and 2) the ROls in visual network not directly stimulated (Group 2, "Indirectly-stimulated visual cortex"). For each of these two ROI groups, the average FC to other Visual module ROls (i.e., 'Within Visual FC') and the average FC to non-Visual ROls (i.e., 'Group $1 / 2$ to Non-visual FC') were measured. Results largely followed similar, but weaker patterns as found in the above paragraph. For directly-stimulated visual cortex (Group 1), the Intensity by Type ANOVA did not produce a significant interaction $\left(F_{3,69}=1.85, p=0.15\right)$, and two separate one-way ANOVA showed no effect of Intensity in either "Group 1 to Visual" $F C\left(F_{3,69}=1.18, p=0.33\right)$ or "Group 1 to Non-Visual" FC $\left(F_{3,69}=0.26\right.$, $p=0.85$ ). In comparison, the results for indirectly-stimulated visual cortex (Group 2) were largely consistent with the results based on the whole Visual network (Figs. 4B, 4C): the Intensity by Type ANOVA revealed a significant Intensity $x$ Type interaction $\left(F_{3,69}=3.12, p=\right.$ 0.032), and two separate one-way ANOVA showed no effect of Intensity in "Group 2 to Visual" $\mathrm{FC}\left(\mathrm{F}_{3,69}=1.65, \mathrm{p}=0.17\right)$ but significant "Group 2 to Non-Visual" $F C\left(F_{3,69}=3.38, p=0.023\right)$. In summary, these ancillary findings demonstrated the distributed nature of TMS effects across the visual system, with changes in FC largely observed in brain regions that were not directly stimulated by the TMS E-field.

\subsubsection{Behavioral correlates of visual system FC}

408 A second goal of this study was to examine the link between FC and performance. Because TMS led to changes in both performance and visual network FC, links between behavior and FC should be expected and may shed light on the neural mechanisms of the TMS effect on performance. In addition, it is possible that within-module FC and between-module FC may relate to performance in different ways, as the effect of TMS intensity has been shown in the previous section to interact with FC types (i.e., within- or between-module connectivity).

414 Therefore, the links between visual system FC and task performance were compared in a mixed effect regression model in which the correct response rate was predicted by the between- 
417 that may affect performance, including TMS intensity, baseline performance, RMT, and gender 418 (see Fig. S1). The effects of these factors on performance were assessed using F-contrasts 419 and T-contrasts and are summarized in Table 1.

Table 1. Effects of TMS Intensity (\%RMT) and FC type on functional connectivity.

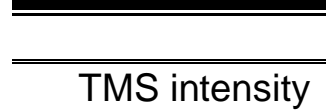

Between-module FC

Within-module FC

Between vs Within-module

Between-module FC x Intensity

Within-module FC x Intensity

Note: $*: \mathrm{p}<0.05, * *: \mathrm{p}<0.01$

\section{Z-stat}

$-3.02 * *$

$2.26 *$

$3.23 * *$

-
F-stat

0.48 (n.s.)

$-$
0.045 (n.s.)

1.82 (n.s.)

In contrast to the behavioral findings in Section 3.1, once FC measures were considered in the model, the TMS intensity showed no reliable effect on performance $\left(F_{3,64.12}=0.48, p=0.70\right)$. In fact, the between-module FC, previously shown to be significantly modulated by TMS, negatively predicted response accuracy (beta $=-0.27, z=-3.02, p=0.0025$, Fig. 5A). The within-module FC, in contrary, positively predicted response accuracy (beta $=0.12, z=2.26, p=$ 0.024 , Fig. 5B). Such differences in behavioral association were further indicated by a Type by FC interaction (i.e., between-module minus within-module; beta $=0.39, z=3.23, p=0.0013$ ), suggesting that the effect of TMS on FC during task performance was dependent on the type of FC. There was no significant Intensity x FC interaction for either within-module $F C\left(F_{3,63.54}=\right.$ $1.82, p=0.15)$ or between-module $F C\left(F_{3,63.84}=0.045, p=0.99\right)$ of the Visual module. In sum, 435 relationships.
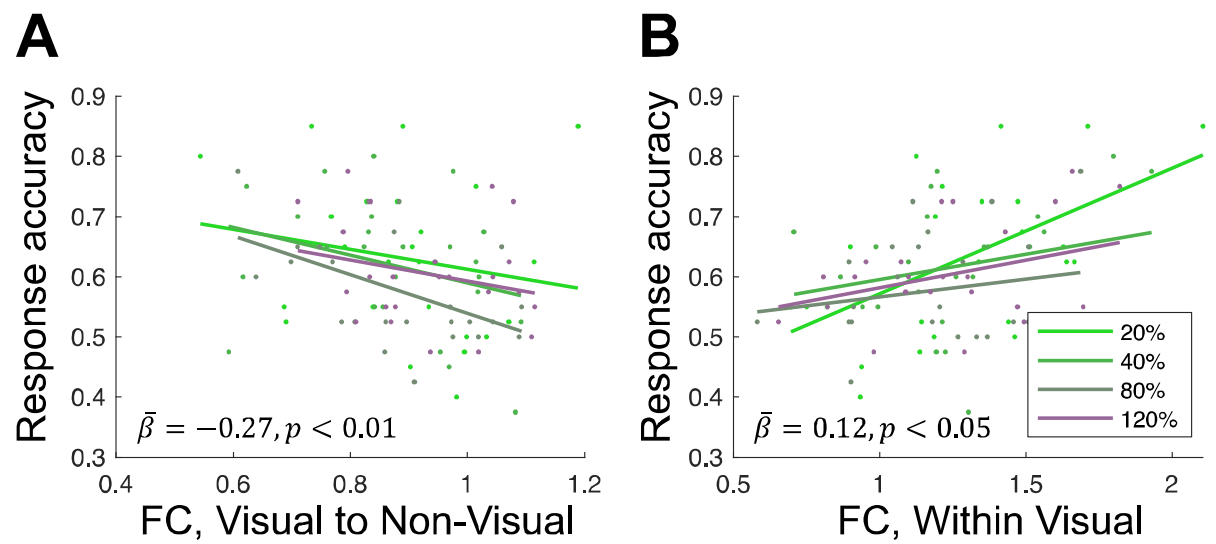
Figure 5. Relationships between task performance and FC either $(\boldsymbol{A})$ between the Visual system and all non-Visual modules, or (B) within the Visual network.

\subsubsection{Structural constraints in TMS-induced functional connectivity}

441 The third goal of this study was to determine if FC patterns, constrained by the structural 442 connectivity (SC) network, can be altered by TMS. As the pattern of functional communication 443 has been shown to rely on a backbone of white-matter connections (Hermundstad et al., 2013; 444 Goni et al., 2014), it was predicted that the impact of TMS on FC can be reflected by the level of 445 consistency between the functional network and its underlying structural network. At the most 446 basic level, FC-SC similarity can be characterized with Spearman's correlation between 447 structural and functional networks; implicit in this relatively straightforward approach is the 448 premise that FC should reflect the underlying SC, and that lower FC-SC similarity indicates a 449 greater deviation of the FC pattern from a more original configuration constrained by the 450 structural backbone, hence reflecting a greater disruption of the FC pattern.

451 For the Visual network, an Intensity by Type repeated measures ANOVA on FC-SC similarity 452 was employed to assess if and how TMS contributes to the change in FC. The analysis 453 revealed a significant effect of Intensity $\left(F_{3,63}=6.17, p=9.6 e-4\right)$, as well as a significant 454 Intensity by Type interaction $\left(F_{3,63}=4.42, p=6.9 e-3\right.$, see Fig. 6A, 6B). Separate one-way 455 repeated measure ANOVAs were performed to further examine how between-module and 456 within-module connections were affected. As shown in Figure 6B, the TMS intensity effect 457 turned out to be significant for within-module FC-SC similarity $\left(F_{3,63}=11.18, p=5.65 e-6\right)$ in the 458 Visual network, leading to more distinct patterns of FC at higher intensity levels. In comparison, 459 TMS intensity showed no effect for between-module FC-SC similarity $\left(F_{3,63}=0.65, p=0.59\right)$.

460 Lastly, an exploratory analysis was performed to test whether the FC pattern between other 461 modules could be affected by TMS (Fig. 6C), which further confirmed that the TMS-induced 462 disruption of FC pattern was specific to the FC within visual system. 


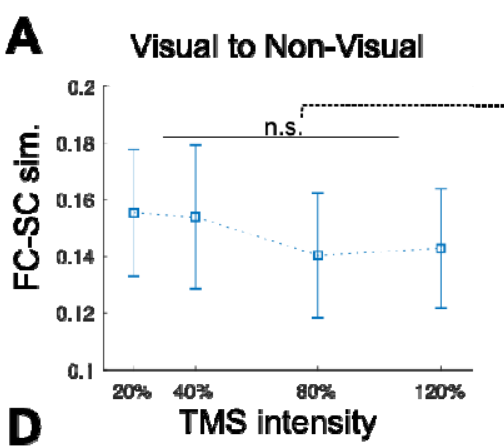

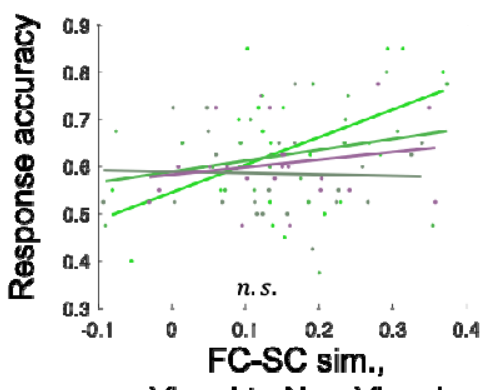

Visual to Non-Visual
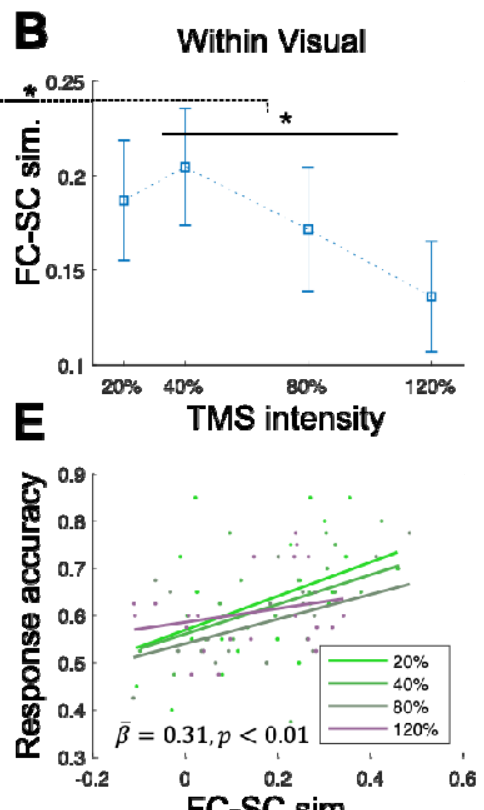

Within Visual

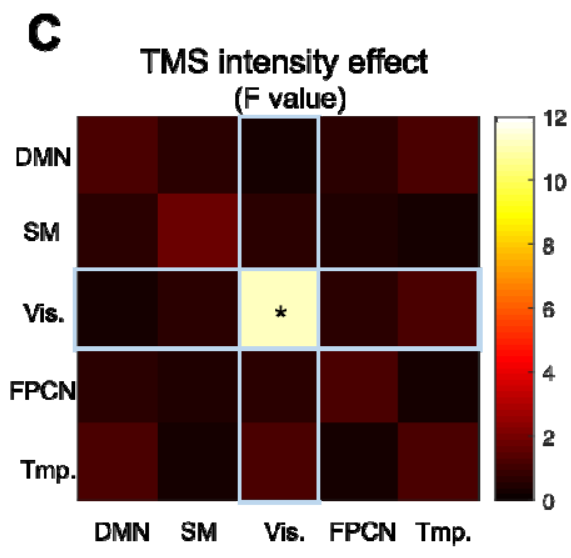

Figure 6. The effects of TMS intensity on visual network FC-SC similarity. Dashed horizontal brace indicates the Intensity $x$ Type interaction; horizontal solid lines indicate main effect of Intensity in the Within Visual (B) connections, but not from Visual to Non-Visual (A) connections. C) The effects of TMS intensity (expressed as F-values) on the patterns of FC (measured as FC-SC similarity) between different modules. (D) and (E) The relationships between task performance and FC-SC similarity. Error bars indicate standard error of the mean (SEM). Asterisks (*) indicate $p<0.05$.

Next, to determine if FC-SC coupling influenced motion perception accuracy a mixed effect model was performed in which the correct response rate for each participant, under each TMS intensity level, was assigned as the outcome variable. The TMS intensity levels and its interactions with the between-module FC-SC similarity and the within-module FC-SC similarity of the visual module were modeled as fixed effect. Potential covariates, including sex, coherence of dots, and RMT of each subject, were included in the model and treated as fixed effect as well. The effects of these factors on performance were assessed using F-contrasts and T-contrasts, and they are summarized in Table 2. Similar to the previous finding in Section 3.3.2, with the brain measure (FC-SC similarity) taken into account, TMS intensity no longer showed a significant effect on the correct response rate $\left(F_{3,56.71}=0.19, p=0.90\right)$. The withinmodule FC-SC similarity of the visual network, previously shown to be modulated by TMS, was positively predicting the correct response rate (beta $=0.31, z=2.71, p=0.0068$, Fig. $6 \mathrm{E}$ ). The between-module FC-SC similarity of the visual module was not significantly related to correct 
effect was found to be trending significant (i.e., between-module minus within-module; beta = $0.36, z=1.77, p=0.077)$.

Table 2. Effects of TMS Intensity (\%RMT) and FC-SC similarity on Behavior.

\begin{tabular}{lcc}
\hline & Z-stat & F-stat \\
\hline TMS intensity & - & 0.19 (n.s.) \\
Between-module FC-SC sim. & -0.32 (n.s.) & - \\
Within-module FC-SC sim. & $2.71 * *$ & - \\
Between vs Within-module & 1.77. & - \\
Between-module FC-SC sim. x Intensity & - & 1.66 (n.s.) \\
Within-module FC-SC sim. x Intensity & - & 0.24 (n.s.) \\
\hline
\end{tabular}

\section{DISCUSSION}

Current views of the neural effects of TMS are changing. Earlier basic science approaches to TMS in humans focused on the effects of localized stimulation to a specific cortical target. As both imaging and TMS techniques evolved, a more recent focus on bivariate connections from the stimulation site to a subcortical target have revealed promising findings, such as the possibility of targeting subcortical structures with cortical TMS (Wang et al., 2014; Luber et al., in press). However, there is a growing appreciation for how stimulation at a single site can propagate throughout a cortical network (Cocchi et al., 2016) and a growing need to identify which principles govern that flow. Though there are only a limited number of combined TMSEEG or TMS-fMRI studies considering this problem from a network-level perspective, one general principle that emerges across studies is that local stimulation spreads according to the functional networks associated with the stimulated node (Momi et al., 2021b). This study advances the field by considering this phenomenon during the engagement of an active task, and finds that the effects of TMS on ongoing brain activity are most evident in regions actively engaged with the task at hand. This is a critical extension of expectations of local cortical excitation or inhibition with specific stimulation frequencies (Aydin-Abidin et al., 2008), and to

508 the variability of modulation observed during different brain states (Gollo et al., 2017). By 509 stimulating with bursts of $10 \mathrm{~Hz}$ rTMS at different intensities over the primary visual cortex 510 during a motion perception task, and concurrent with fMRI imaging, the present study revealed 511 four novel findings. First, motion processing was selectively impaired at $80 \%$ RMT. Second, the 512 connectivity between the visual system and other cortical communities increased with 
513 stimulation intensity. Third, intra-community connectivity was beneficial, while the extra-

514 community connectivity induced by TMS was detrimental to performance. Lastly, structural

515 connectivity constrained this effect, such that FC-SC was strongest in low-intensity conditions,

516 and was disrupted with increasing intensity, resulting in poorer performance. These findings are

517 discussed in greater detail below.

518 Before turning to the connectivity-related findings, it is important to consider the 519 observation that TMS-related impairment of motion discrimination was selective to the $80 \%$ 520 RMT condition. No differences in discrimination accuracy were seen between the highest $(120 \%$ 521 RMT) and lowest (20\% RMT) stimulation intensities, suggesting that the disruptive effects were 522 attributable to a neural response in visual cortex, and not distraction-related confounds 523 associated with increasing TMS intensity (e.g., noise, cutaneous sensation). Past studies have 524 reported a wide variety of behavioral effects that result from single or paired pulse TMS over a 525 range of factors, such as timing, intensity, and the ongoing activation state of the stimulated region. For example, in primary motor cortex the threshold for inhibitory effects of TMS is approximately $80 \%$ of RMT (Kallioniemi et al., 2014). Thus, TMS intensity can determine the local balance between inhibitory and excitatory circuit recruitment. In another example, single pulses of $80 \%$ RMT TMS timed to the onset of the N2 latency of MT+ facilitate motion perception (Gamboa Arana et al., 2020). In addition to the possibility of a specific balance of inhibitory and excitatory effects, such findings may be explained by a stochastic resonance account, such that the strength of a stimulus can be improved by externally enhancing the ongoing neural activity. Despite a similar task paradigm and dosing structure, the current study instead revealed a disruption of performance at $80 \% \mathrm{RMT}$, perhaps due to the differences in cortical target (V1 vs. MT+), or the use of three pulses (spaced at $10 \mathrm{~Hz}$ ) instead of one pulse of TMS. Furthermore, this single pulse was timed to the onset of a specific, motion sensitive ERP. Nonetheless, the current results may abide by the principle that a selective modulation of performance is based on the engagement with ongoing brain activity, such that that $10 \mathrm{~Hz}$ pulses timed to the onset of motion disrupted the downstream flow of information from primary visual cortex to motion-sensitive cortex $(\mathrm{MT}+)$. This pattern of results also lends support to a 541 more global model of the effects of TMS on behavior (Silvanto and Cattaneo, 2021) that posits 542 distinct facilitatory and suppressive ranges as a function of TMS intensity, and which are shifted 543 by state-dependent changes in neural excitability (e.g., during stimulus processing vs. rest 544 states).

$545 \quad$ Turning to the general connectivity changes in response to TMS, the current study first 546 found that local connectivity within the visual system was more resilient to stimulation intensity 
547 than global connectivity from the visual system to the rest of the brain. Such a result is 548 consistent with the notion that the network responsible for visual motion is an optimized system 549 that operates best when allowed to segregate from other cortical networks. In contrast, when 550 stimulation intensity induced an increased spread of connectivity between modules, this more 551 global pattern had negative consequences for behavior. In fact, when we examined the source 552 of these intensity-related increases in between-module FC, we found greater FC with the 553 somatosensory-motor and temporal networks, consistent with the idea that increasing intensity 554 of TMS elicited greater network activity due to increasing motor (SM) or auditory (Temporal) 555 sensation. Thus, an important consideration for studies examining the network-level effects of 556 TMS is to consider how some of these distant effects can be attributed to the sensory properties 557 of TMS stimulation, as revealed by conditions that mimic the sound and somatosensory 558 attributes of TMS while minimizing intracranial E-fields ("sham" conditions). In some cases, 559 these confounds may be unavoidable (see Conde et al., 2019 for innovative post-processing techniques) but they are not necessarily a barrier to interpreting effects in non-sensorial regions.

An interesting, if overlooked, observation in many concurrent TMS-fMRI studies is that the effects of localized TMS are not strongest at the site of stimulation and are often seen in distant regions of the cortex. While some of these distant effects can be attributed to the sensory properties of TMS stimulation, discussed above, a recent meta-analysis revealed that this surprising absence of a local effect generalizes across many different stimulation paradigms (Rafiei and Rahnev, 2021). More broadly, it is important to consider how such networks may be influenced or modulated by the level of attention associated with increasing TMS intensity. Selective attention can modulate the ongoing processing in both visual (Ballesteros et al., 2008) and motor (Johansen-Berg and Matthews, 2002) regions, and thus it is possible that heightened responses in sensory regions reflect an increase in the alerting or arousal effect associated with increasing TMS intensity, in addition to the input from more basic sensory afferents. While not

572 an explicit focus of this study, such an interpretation is not inconsistent with the finding that 573 attention-driven MT+ activity during motion-perception tasks is influenced by the gating of V1

574 and V5 connectivity by the posterior parietal cortex (Silvanto et al., 2005). Taken together, this

575 perspective links how a network-level interpretation of TMS effects can better explain the 576 function of single region in a global perspective. While there are many studies on the impact of 577 TMS on local function of the visual regions during motion paradigms (Campana et al., 2002; 578 Cowey et al., 2006; Amemiya et al., 2017), there is minimal research on the network-based 579 influence on this region. 
Another important finding was that connectivity within the visual network was positively associated with motion accuracy, whereas the connectivity between visual and other networks was negatively associated motion accuracy. Furthermore, follow-up tests demonstrated that the positive correlation between within-module FC and response accuracy was strongest at the lowest TMS intensity, when our exogenous stimulation was unlikely to have much of an effect on cortical excitability. In contrast, increasingly worse performance was associated with more global connectivity at higher levels of TMS intensity (Table 1). Such a benefit to more segregated cortical networks is well known in developmental (Stevens et al., 2012) and aging (Betzel et al., 2014; Crowell et al., 2020) literatures, but this is the first finding to suggest an active, parametric perturbation of cortical modularity and its consequences for behavior. An alternative explanation for these effects is that greater stimulation intensity also activated auditory and somatosensory-motor regions, and that this influence of non-visual systems resulted in more interference to the efficient processing of visual stimuli. However, this view is not supported by the fact that motion discrimination performance was greater at $120 \%$ RMT than $80 \%$ RMT, suggesting a non-linear relationship between delivered E-field and its effect on behavior in this system.

Lastly, an analysis of SC data revealed that the propagation of these effects was largely constrained by multiple indices of white matter anatomy. Two related findings support the notion that the FC patterns associated with localized TMS stimulation are constrained by structural architecture. First, we examined how FC-SC correlations change as a function of TMS intensity. Collectively, significant effects of both Intensity and a significant Intensity $\times$ FC Type interaction, as well as the decline in within-module connectivity with increasing intensity all suggest that TMS disrupted "normal" visual network functioning and introducing exogenous stimulation disrupted successful motion processing by decoupling FC and SC patterns. Second, and supporting this inference, an Intensity $\times$ FC Type Repeated Measures ANOVA revealed a pattern that mirrors the FC findings above, such that the coupling between structural and FC predicted task performance for the local within-module regions, but more global betweenmodule couplings had no influence on motion perception (Table 2). In our study the FC-SC coupling ranged between $r=[0.0-0.3]$, consistent with other many other investigations between functional and structural network data relying on one-to-one correspondence between FC and 611 suggests that the upper bound of most FC-SC correlations is not very high (Suarez et al., 2020).

612 Future approaches to characterizing FS-SC relationships may consider the value of derived 613 properties of individual nodes that describe their global influence, including modal 
614 controllability - which purports to describe the energy associated with transitioning between 615 brain states (Gu et al., 2017; Beynel et al., 2020b).

616 While the current study represents a novel application of concurrent TMS-fMRI to 617 answer unexplained phenomena describing the spread of focal neurostimulation across the 618 cortex, several limitations were encountered in the pursuit of this goal. First, the study design 619 did not incorporate sham conditions that closely replicate the sound and scalp sensation of TMS 620 without the delivery of significant E-field to the brain. Nevertheless, the results of the parametric 621 investigation of TMS intensity at the target support a cortical origin of the stimulation effects.

622 Second, because the goal of the current study was to investigate multiple stimulation intensities, 623 it was not feasible to complete within a typical MRI scanning session to assess a 624 complementary stimulation site to examine the potential dissociations between task-relevant 625 and task-irrelevant cortical sites. Lastly, the current design relied on a trial-related timing of 10 $626 \mathrm{~Hz}$ pulses concurrent with the task; these design choices represent only one of a number of 627 parameters for frequency, number of pulses, and timing relative to the motion perception task. 628 While the prolonged effects of rTMS are generally assumed to relate to synaptic plasticity, the 629 detailed effects of stimulation on a region's excitability via long-term depression or potentiation 630 remains a matter of investigation.

The observed pattern of findings suggests that inducing a more global pattern of connectivity with higher intensity TMS contributes to disruptions in visual motion perception. Our results show that this spread is largely limited to the network of cortical sites involved in the active task (i.e., visual motion), and that TMS-related disruptions of this network are detrimental to cognitive performance. Lastly, we find that the functional connectivity spread with increasing TMS is largely constrained by structural connectivity, suggesting that the use of structural network information can help to predict at least some of the influence of local perturbations on a more global cortical network. Thus, while it is widely known that TMS may disrupt cognition, these results help us to explain how TMS disrupts local and global networks to induce perceptual change.

Alekseichuk I, Mantell K, Shirinpour S, Opitz A (2019) Comparative modeling of transcranial magnetic and electric stimulation in mouse, monkey, and human. Neuroimage 194:136148.

Amemiya T, Beck B, Walsh V, Gomi H, Haggard P (2017) Visual area V5/hMT+ contributes to perception of tactile motion direction: a TMS study. Sci Rep 7:40937. 
648

649

650

651

652

653

654

655

656

657

658

659

660

661

662

663

664

665

666

667

668

669

670

671

672

673

674

675

676

677

678

679

680

681

682

683

684

685

686

687

688

689

690

691

692

693

694

695

696

697

698

Aydin-Abidin S, Trippe J, Funke K, Eysel UT, Benali A (2008) High- and low-frequency repetitive transcranial magnetic stimulation differentially activates c-Fos and zif268 protein expression in the rat brain. Experimental brain research 188:249-261.

Ballesteros S, Reales JM, Mayas J, Heller MA (2008) Selective attention modulates visual and haptic repetition priming: effects in aging and Alzheimer's disease. Experimental brain research 189:473-483.

Betzel RF, Byrge L, He Y, Goni J, Zuo XN, Sporns O (2014) Changes in structural and functional connectivity among resting-state networks across the human lifespan. Neuroimage 102 Pt 2:345-357.

Beynel L, Powers JP, Appelbaum LG (2020a) Effects of repetitive transcranial magnetic stimulation on resting-state connectivity: A systematic review. Neuroimage:116596.

Beynel L, Davis S, Crowell C, Hilbig S, Lim W, Nguyen D, Palmer H, Brito A, Peterchev A, Luber B (2019) Online repetitive transcranial magnetic stimulation during working memory in younger and older adults: A randomized within-subject comparison. PloS one 14:e0213707.

Beynel L, Deng L, Crowell CA, Dannhauer M, Palmer H, Hilbig S, Peterchev AV, Luber B, Lisanby SH, Cabeza R, Appelbaum LG, Davis SW (2020b) Structural Controllability Predicts Functional Patterns and Brain Stimulation Benefits Associated with Working Memory. The Journal of neuroscience : the official journal of the Society for Neuroscience 40:6770-6778.

Beynel L, Davis SW, Crowell CA, Dannhauer M, Lim W, Palmer H, Hilbig SA, Brito A, Hile C, Luber B, Lisanby SH, Peterchev AV, Cabeza R, Appelbaum LG (2020c) Site-Specific Effects of Online rTMS during a Working Memory Task in Healthy Older Adults. Brain Sci 10.

Beynel L, Dannhauer M, Palmer H, Hilbig SA, Crowell CA, Wang JE, Michael AM, Wood EA, Luber B, Lisanby SH, Peterchev AV, Cabeza R, Davis SW, Appelbaum LG (2021) Network-based rTMS to modulate working memory: The difficult choice of effective parameters for online interventions. Brain Behav 11:e2361.

Brascamp JW, Kanai R, Walsh V, van Ee R (2010) Human middle temporal cortex, perceptual bias, and perceptual memory for ambiguous three-dimensional motion. $\mathrm{J}$ Neurosci 30:760-766.

Campana G, Cowey A, Walsh V (2002) Priming of motion direction and area V5/MT: a test of perceptual memory. Cereb Cortex 12:663-669.

Castrillon G, Sollmann N, Kurcyus K, Razi A, Krieg SM, Riedl V (2020) The physiological effects of noninvasive brain stimulation fundamentally differ across the human cortex. Sci Adv 6:eaay2739.

Cocchi L, Sale MV, L LG, Bell PT, Nguyen VT, Zalesky A, Breakspear M, Mattingley JB (2016) A hierarchy of timescales explains distinct effects of local inhibition of primary visual cortex and frontal eye fields. Elife 5.

Conde V, Tomasevic L, Akopian I, Stanek K, Saturnino GB, Thielscher A, Bergmann TO, Siebner HR (2019) The non-transcranial TMS-evoked potential is an inherent source of ambiguity in TMS-EEG studies. Neuroimage 185:300-312.

Conforto AB, Z'Graggen WJ, Kohl AS, Rosler KM, Kaelin-Lang A (2004) Impact of coil position and electrophysiological monitoring on determination of motor thresholds to transcranial magnetic stimulation. Clinical neurophysiology : official journal of the International Federation of Clinical Neurophysiology 115:812-819.

Cowey A, Campana G, Walsh V, Vaina LM (2006) The role of human extra-striate visual areas V5/MT and V2/V3 in the perception of the direction of global motion: a transcranial magnetic stimulation study. Experimental brain research 171:558-562.

Crowell CA, Davis SW, Beynel L, Deng L, Lakhlani D, Hilbig SA, Palmer H, Brito A, Peterchev AV, Luber B, Lisanby SH, Appelbaum LG, Cabeza R (2020) Older adults benefit from 
699

700

701

702

703

704

705

706

707

708

709

710

711

712

713

714

715

716

717

718

719

720

721

722

723

724

725

726

727

728

729

730

731

732

733

734

735

736

737

738

739

740

741

742

743

744

745

746

747

748

more widespread brain network integration during working memory. Neuroimage 218:116959.

Davis SW, Szymanski A, Boms H, Fink T, Cabeza R (2019) Cooperative contributions of structural and functional connectivity to successful memory in aging. Netw Neurosci 3:173-194.

Fornito A, Zalesky A, Bullmore ET (2010) Network scaling effects in graph analytic studies of human resting-state FMRI data. Front Syst Neurosci 4:22.

Gamboa Arana OL, Palmer H, Dannhauer M, Hile C, Liu S, Hamdan R, Brito A, Cabeza R, Davis SW, Peterchev AV, Sommer MA, Appelbaum LG (2020) Intensity- and timingdependent modulation of motion perception with transcranial magnetic stimulation of visual cortex. Neuropsychologia 147:107581.

Gamboa OL, Brito A, Abzug Z, D'Arbeloff T, Beynel L, Wing EA, Dannhauer M, Palmer H, Hilbig SA, Crowell CA, Liu S, Donaldson R, Cabeza R, Davis SW, Peterchev AV, Sommer MA, Appelbaum LG (2020) Application of long-interval paired-pulse transcranial magnetic stimulation to motion-sensitive visual cortex does not lead to changes in motion discrimination. Neuroscience letters 730:135022.

Gollo LL, Roberts JA, Cocchi L (2017) Mapping how local perturbations influence systems-level brain dynamics. Neuroimage.

Gomez LJ, Dannhauer M, Koponen LM, Peterchev AV (2020) Conditions for numerically accurate TMS electric field simulation. Brain stimulation 13:157-166.

Goni J, van den Heuvel MP, Avena-Koenigsberger A, Velez de Mendizabal N, Betzel RF, Griffa A, Hagmann P, Corominas-Murtra B, Thiran JP, Sporns O (2014) Resting-brain functional connectivity predicted by analytic measures of network communication. Proc Natl Acad Sci U S A 111:833-838.

Gu S, Betzel RF, Mattar MG, Cieslak M, Delio PR, Grafton ST, Pasqualetti F, Bassett DS (2017) Optimal trajectories of brain state transitions. Neuroimage 148:305-317.

Gullmar D, Haueisen J, Reichenbach JR (2010) Influence of anisotropic electrical conductivity in white matter tissue on the EEG/MEG forward and inverse solution. A high-resolution whole head simulation study. Neuroimage 51:145-163.

Hermundstad AM, Bassett DS, Brown KS, Aminoff EM, Clewett D, Freeman S, Frithsen A, Johnson A, Tipper CM, Miller MB, Grafton ST, Carlson JM (2013) Structural foundations of resting-state and task-based functional connectivity in the human brain. Proc Natl Acad Sci U S A 110:6169-6174.

Honey CJ, Kotter R, Breakspear M, Sporns O (2007) Network structure of cerebral cortex shapes functional connectivity on multiple time scales. Proceedings of the National Academy of Sciences of the United States of America 104:10240-10245.

Horn A, Ostwald D, Reisert M, Blankenburg F (2014) The structural-functional connectome and the default mode network of the human brain. Neuroimage $102 \mathrm{Pt}$ 1:142-151.

Ishikawa K, Nagai T, Yamada Y, Negi A, Nakamura M (2011) Optimal conditions for multifocal VEP recording for normal Japanese population established by receiver operating characteristic analysis. Doc Ophthalmol 122:29-37.

Johansen-Berg H, Matthews PM (2002) Attention to movement modulates activity in sensorimotor areas, including primary motor cortex. Experimental brain research 142:13-24.

Kallioniemi E, Saisanen L, Kononen M, Awiszus F, Julkunen P (2014) On the estimation of silent period thresholds in transcranial magnetic stimulation. Clinical neurophysiology : official journal of the International Federation of Clinical Neurophysiology 125:22472252.

Luber B, Deng Z-D (2016) Application of non-invasive brain stimulation in psychophysiology. In: Handbook of Psychophysiology (Berntson GG, Cacioppo JT, Tassinary LG, eds), pp 116-150. Cambridge: Cambridge University Press. 
Luber B, Davis SW, Deng Z-D, Murphy D, Peterchev AV, Lisanby SH (in press) Using diffusion tensor imaging to effectively target TMS to deep brain structures. Neurolmage.

Momi D, Ozdemir RA, Tadayon E, Boucher P, Shafi MM, Pascual-Leone A, Santarnecchi E (2021a) Network-level macroscale structural connectivity predicts propagation of transcranial magnetic stimulation. Neuroimage 229:117698.

Momi D, Ozdemir RA, Tadayon E, Boucher P, Di Domenico A, Fasolo M, Shafi MM, PascualLeone A, Santarnecchi E (2021b) Perturbation of resting-state network nodes preferentially propagates to structurally rather than functionally connected regions. Sci Rep 11:12458.

Ozdemir RA, Tadayon E, Boucher P, Momi D, Karakhanyan KA, Fox MD, Halko MA, PascualLeone A, Shafi MM, Santarnecchi E (2020) Individualized perturbation of the human connectome reveals reproducible biomarkers of network dynamics relevant to cognition. Proc Natl Acad Sci U S A 117:8115-8125.

Peterchev AV, Wagner TA, Miranda PC, Nitsche MA, Paulus W, Lisanby SH, Pascual-Leone A, Bikson M (2012) Fundamentals of transcranial electric and magnetic stimulation dose: definition, selection, and reporting practices. Brain stimulation 5:435-453.

Rafiei F, Rahnev D (2021) Does TMS increase BOLD activity at the site of stimulation? [Preprint].

Reichardt J, Bornholdt S (2006) Statistical mechanics of community detection. Phys Rev E Stat Nonlin Soft Matter Phys 74:016110.

Sanders LL, Auksztulewicz R, Hohlefeld FU, Busch NA, Sterzer P (2014) The influence of spontaneous brain oscillations on apparent motion perception. Neuroimage $102 \mathrm{Pt}$ 2:241-248.

Silvanto J, Cattaneo Z (2021) Nonlinear interaction between stimulation intensity and initial brain state: Evidence for the facilitatory/suppressive range model of online TMS effects. Neuroscience letters 742:135538.

Silvanto J, Lavie N, Walsh V (2005) Double dissociation of V1 and V5/MT activity in visual awareness. Cerebral cortex 15:1736-1741.

Silvanto J, Bona S, Marelli M, Cattaneo Z (2018) On the Mechanisms of Transcranial Magnetic Stimulation (TMS): How Brain State and Baseline Performance Level Determine Behavioral Effects of TMS. Frontiers in psychology 9:741.

Smith RE, Tournier JD, Calamante F, Connelly A (2013) SIFT: Spherical-deconvolution informed filtering of tractograms. Neuroimage 67:298-312.

Stephan KE, Kasper L, Harrison LM, Daunizeau J, den Ouden HE, Breakspear M, Friston KJ (2008) Nonlinear dynamic causal models for fMRI. Neuroimage 42:649-662.

Stevens AA, Tappon SC, Garg A, Fair DA (2012) Functional brain network modularity captures inter- and intra-individual variation in working memory capacity. PloS one 7:e30468.

Suarez LE, Markello RD, Betzel RF, Misic B (2020) Linking Structure and Function in Macroscale Brain Networks. Trends Cogn Sci 24:302-315.

Thielscher A, Antunes A, Saturnino GB (2015) Field modeling for transcranial magnetic stimulation: a useful tool to understand the physiological effects of TMS? leee Eng Med Bio:222-225.

van den Heuvel MP, Mandl RC, Kahn RS, Hulshoff Pol HE (2009) Functionally linked restingstate networks reflect the underlying structural connectivity architecture of the human brain. Hum Brain Mapp 30:3127-3141.

Wang JX, Voss JL (2015) Long-lasting enhancements of memory and hippocampal-cortical functional connectivity following multiple-day targeted noninvasive stimulation. Hippocampus 25:877-883.

Wang JX, Rogers LM, Gross EZ, Ryals AJ, Dokucu ME, Brandstatt KL, Hermiller MS, Voss JL (2014) Targeted enhancement of cortical-hippocampal brain networks and associative memory. Science 345:1054-1057. 
bioRxiv preprint doi: https://doi.org/10.1101/2022.01.06.475293; this version posted January 10, 2022. The copyright holder for this preprint (which was not certified by peer review) is the author/funder. All rights reserved. No reuse allowed without permission.

800

Zinchenko A, Brunner S, Chen L, Shi Z, Taylor PCJ, Muller HJ (2021) V5/MT+ modulates

801

802 spatio-temporal integration differently across and within hemifields: Causal evidence

803 from TMS. Neuropsychologia 161:107995. 